\title{
Comparison of Different Image Compression Techniques
}

\author{
Afshan Mulla \\ Namrata Gunjikar ${ }^{\S}$ \\ Students ${ }^{\S}$, Sardar Patel Institute of Technology, \\ Bhartiya Vidya Bhavans campus, Andheri (W).
}

Radhika Naik ${ }^{\S}$

\begin{abstract}
In recent years, the development and demand of multimedia product is growing increasingly fast, contributing to insufficient bandwidth of network and storage of memory device. This justifies the use of different compression techniques to decrease the storage space and efficiency of transferring the images over the network to conserve the bandwidth. A quality constrained compression algorithm based on Discrete Wavelet Transform (DWT) having a spatial-frequency decomposition property which provides quality assessment for images is implemented. 3D spiral JPEG based lossy image compression method which was implemented is based on 3-dimensional formation of the original image by spiral order scanning and 3D Discrete Cosine Transform (DCT). An application of optimum vector quantization on image compression is studied in the Novel $\mathrm{K}$ means algorithm which is similar to the LBG (Linde-BuzoGray) algorithm which provides an optimum codebook for training sequence which will minimize the average distortion over the training sequence, provided certain regularity conditions are satisfied is also evaluated. These image compression techniques are studied and compared for their performance. The results show that the ideal approach for compression of images depends on the type of image that is being compressed. This paper summarizes the different compression methods as it is necessary to reduce the amount of data needed for storage and transmission of information on the basis of different image parameters such as MSE, PSNR etc.
\end{abstract}

General Terms: Transforms, Algorithm, compression, quantization.

Keywords: DWT, spatial-frequency, DCT, code-book, LBG, K-means, MSE, PSNR.

\section{INTRODUCTION}

High quality image processing has become an imperative part in countless dexterous and customer applications. Hence in current day scenario, compression of these High quality images is of great significance in multimedia field. Image compression is the appliance of data compression on digital images which are made up of large number of pixels. In effect, the purpose is to lessen redundancy of the image data in order to store or transmit data in a more efficient form. Compression is a process by which the depiction of computerized information is modified so that the competence necessary for storing or the bit-rate required for transmitting it is reduced. Since image files not only occupy storage but also take up a large portion of bandwidth during transmission, the process of compressing images has become a

necessity. Compression reduces the utilization of exclusive resources such as hard disk space or communication bandwidth.
Therefore, the theory of data compression becomes more and more noteworthy for reducing the data redundancy to save more hardware space and transmission bandwidth. In computer science and information theory, data compression or source coding is the process of encoding information using fewer bits or other information-bearing units than an unencoded representation.

There are two types of compression techniques namely Lossy and Lossless compression. Lossless compression is generally used in medical images, genuine signature and where quality degradation cannot be tolerated. In contrast, lossy compression can achieve very high compression ratio and has wide application in multimedia fields and where quality can be compromised due to human visual perception feature. The secret lies in the fact that the pixel values can be represented in values estimated to the original pixel values. Since human eyes cannot easily distinguish these fine dissimilarities, it embodies the tradeoff between precision and space.

Compression of image data without a huge amount of loss in visual information is mainly due to three reasons. First, images contain a high degree of spatial redundancy because correlation does exist between neighboring pixels. Secondly, they contain some spectral redundancy because of correlation among the chrominance information contained in the color components. Finally, they exhibit some degree of visual redundancy due to human being's visual system and the way humans perceive objects. From the hypothetical point of view, a high compression ratio should be obtained as possible based on the redundancy information mentioned above.

Compression is carried out for the subsequent reasons so as to reduce the processing time and transmission duration. Image compression is minimizing the size in bytes of a graphics file with or without degrading the quality of image within threshold limitations.

\section{DWT Textured Image METHOD}

Discrete Wavelet Transform (DWT) [4] has gained widespread acceptance in signal processing and image compression because it provides an extremely flexible multi-resolution image and can decompose an original image into different sub band images including low- and high- frequencies [1]. The high frequency components provides with the information about the fine edges of the image which is very constructive for recovering the original image at the receiver end.

A color (RGB) image when transmitted acquires more bandwidth and more transmission time. Therefore, address this shortcoming, color images can be represented in monochrome as they require lesser bandwidth and less transmission time. The color information of these monochrome images are then retrieved at the receiver. Once the color graphic is converted to monochrome, sometimes the contrasting colors are mapped to the same gray level as each color in grayscale varies from 0-255 and so their visual difference vanishes. Hence the problem is 
how to convert colors to black and white such that different colors would look unique, even if they have the same luminance component.

The proposed DWT method maps these gray scaled colors to unique textures that are different for different colors. However, instead of having a dictionary of textures and colors, it produces a continuum of textures [6] that naturally toggle between patterns without causing visual imbalance. For this purpose, use of the discrete wavelet transform or DWT, which decomposes an image into several sub bands, each representing different spatial frequency contents has to be used.

\subsection{RGB to Textured Mapping and Encoding:}

\section{Step1:}

The original RGB image is converted to $\mathrm{YCbCr}$ color space so that the Discrete Wavelet transform can be applied to the luminance $(\mathrm{Y})$ component which is shown in Figure 2.1.

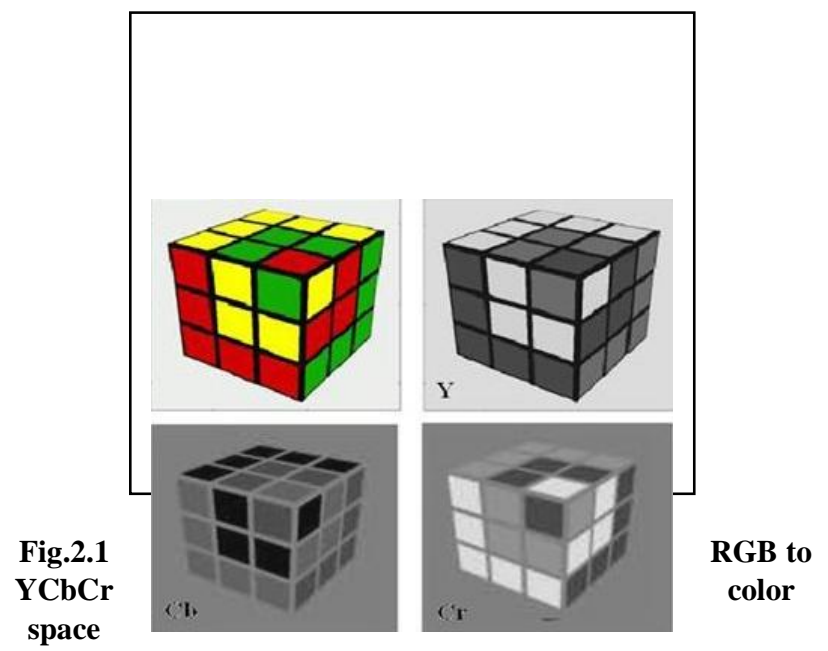

\section{Step2:}

The vertical and horizontal sub-bands are replaced by the $\mathrm{Cb} \&$ $\mathrm{Cr}$ components so that the original color information can be retrieved easily at the receiver side. Then the image containing the low pass sub-band, diagonal sub-band information and $\mathrm{Cb}$ \& $\mathrm{Cr}$ component information is processed further. The inverse DWT is applied to it in order to achieve a textured image. This textured image is finally transmitted over the network which occupies lesser bandwidth and transmission time thereby contributing to the compression of the original image.

The textures [6] incorporated in the image are unique for each and every color and hence when this image is decompressed and decoding at the receiver end the colors can easily be distinguished from each other. This helps in recovering the original RGB image with an acceptable amount of quality degradation due to the compression process executed on the image. The process is illustrated in the figure 2.2. Also this process is reversible and original RGB image can be recovered.

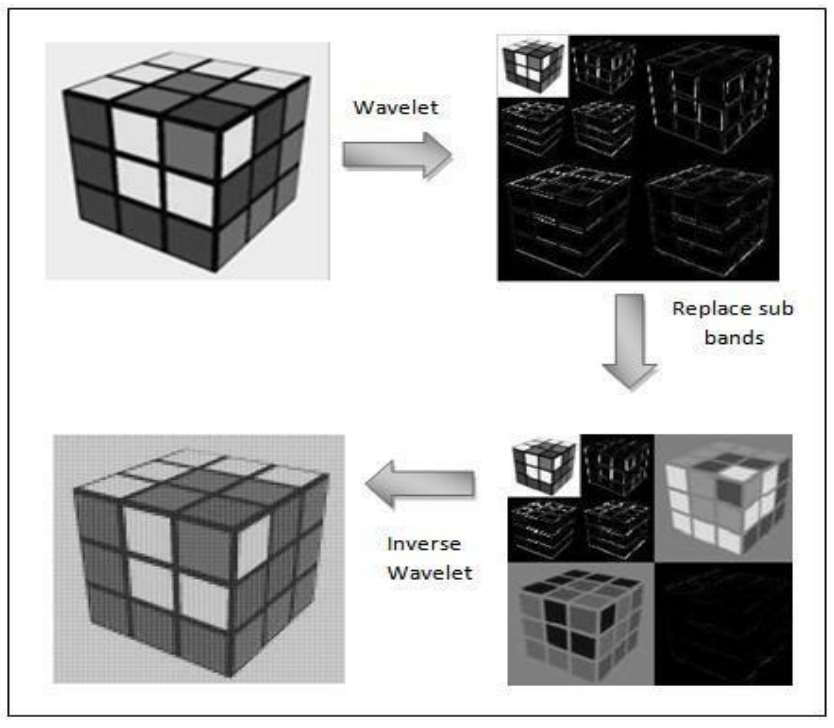

Fig.2.2 Process illustrating Encoding

\subsection{Recovering color image using DWT and Decoding:}

Step1: The textured image received at the recipient side is subjected to DWT transform which divides it into different subbands containing the chrominance $(\mathrm{Cb}$ and $\mathrm{Cr})$ components.

Step2: As seen in the figure 2.3 the image is interpolated and an Inverse DWT is applied in order to get back the luminance $(\mathrm{Y})$ component.

Step3: The luminance as well as the chrominance information is then summed up to recuperate the original RGB image.

The entire process is illustrated in Figure 2.3.

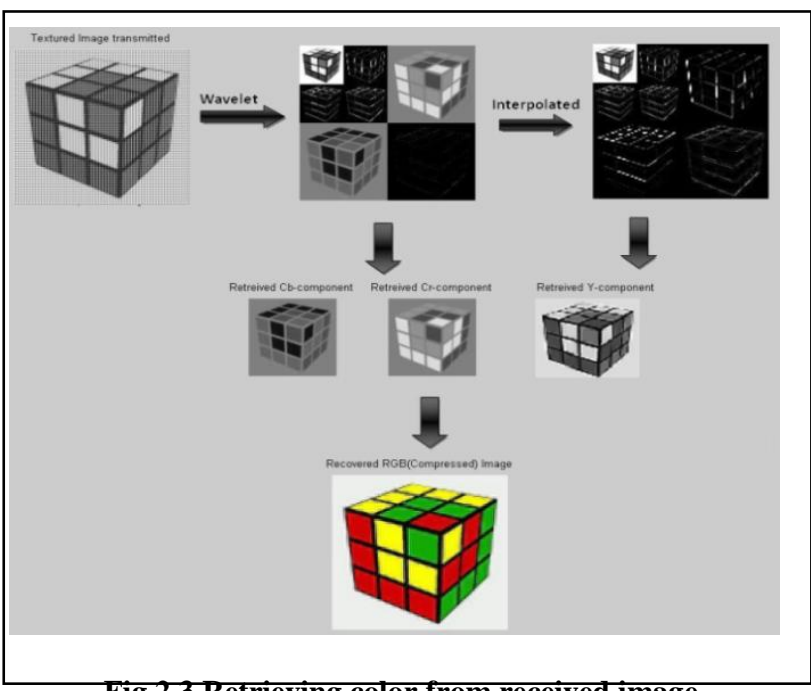

Fig.2.3 Retrieving color from received image

This method is very advantageous to both pictures as well as graphics because of its soft and normal color amalgamation. It is reversible process, enabling repossession of the colors from the textured image. 


\section{K-means ALGORITHM:}

$\mathrm{K}$-means algorithm is a form of vector quantization [5] which makes the use of K-means clustering in which a set of vectors are taken as input. It is a grouping method of bunching analysis which aims at partitioning ' $n$ ' groups into ' $k$ ' clusters in which each group of pixels belongs to the cluster with the nearest mean. A similar method is that of LBG Technique which also comes under Vector Quantization. Also the algorithm of LBG technique [3] is quite similar to the below explained K-means algorithm.

\subsection{Algorithm}

The K-means technique for Image compression steps are as given below:

1. Select a certain number of pixels to be selected for forming a code book (CB).

2. Scan the entire image and sort the pixels into any one of the formed code book on basis of the nearest mean value basis.

3. Scan the image and sort the pixels into the code vectors depending upon the minimum error determined from the previous step.

4. Replace the pixels with the code vector to which they belong thus implementing the quantized algorithm efficiently.

The projected method is an inventive approach to improve the implementation of K-means algorithm. It adopts splitting and sorting policy by separating the input data into groups and applies K-means clustering. K-means segments input image into $\mathrm{K}$ clusters and works in two phases as follows:

1. In the first phase, the input vectors are divided into $\mathrm{N}$ groups based on the mean value of each vector.

2. As the range of the value of a pixel $R$ in image data is $0-255$, the range of the field of each group is set as the ratio of $\mathrm{R}$ to the number of classes $(\mathrm{N})$.

3. A guidance vector is allotted to a class CI, if its mean value is less than or equal to the field of CI. This mean based assemblage of input data speeds up union ensuing in diminution of calculation time.

4. K-Means algorithm is performed on each class of vectors with preliminary seeds. The number of primary seeds ' $M$ ' in each set is relative to its size. The original seeds are chosen with the right intermingle of numerical features (variance, mean, median and mode) of the group inhabitants.

5. The code vectors produced from each category are applied to pruning based on a smallest amount of (Euclidean) distance edge ' $\mathrm{D}$ ' with the code vectors of the incompletely generated codebook.

6. A new code vector 'V1(i)' of a set C(i), will be supplementary to the codebook only if its Euclidean distance with all of the code vectors of the groups from $\mathrm{C} 1$ to $\mathrm{Ci}-1$ is superior than a predefined threshold ' $D$ '.

7. Thus, pruning of code vectors are done at the assembly phase which appreciably reduces the code book size and computational time as well.
K-means algorithm is used for compressing images for its features such as speed, good compression ratio and efficient quality based compression. It is often used as an intermediate step for other algorithms. The flowchart for the same in illustrated in fig 3.1

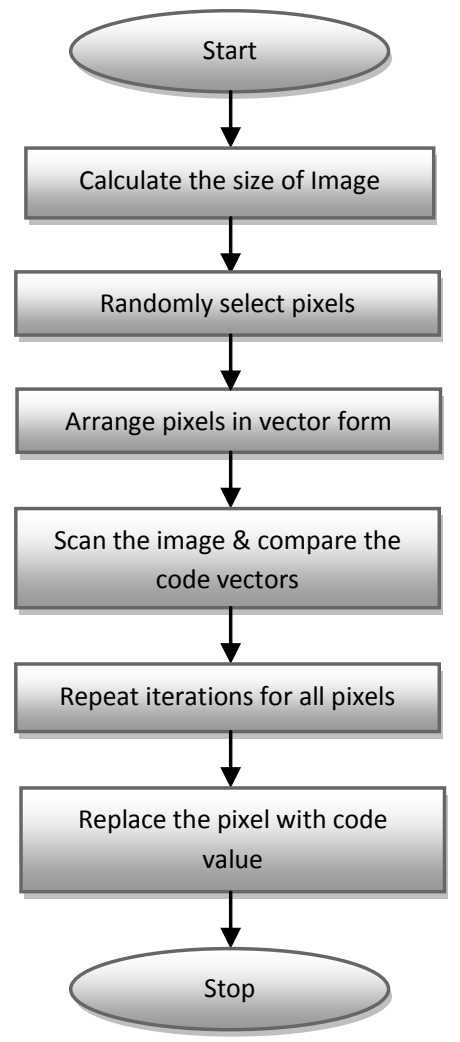

Fig.3.1 Flowchart for K-means Technique

\section{SPIRAL 3D JPEG SCHEME}

The proposed compression scheme is a novel JPEG-based lossy image compression method based on 3-D arrangement of the original image by coiled array scanning [2] and 3D Discrete Cosine Transformation (DCT). Discrete Cosine Transform (DCT) [4] is broadly used transform method in image compression techniques. 2D-DCT is basically used in image compression because image data are by default two dimensional in nature. In general, escalating the dimension of any system generates opportunities for obtaining secondary distances in new multi-dimensional collection. Arrangement of 2D image in the form of 3D cubes is a way to get enhanced assembly of image blocks. However, this entire process should result into a 3-D image which is similar to the original 2-D image.

The conversion of 2D images into 3D images helps us perform the zigzag scanning and quantization on each of the three axes thereby contributing to better compression quality and serving the need for compression in a more evocative way. Hence performing all the steps on the 3D image rather than a $2 \mathrm{D}$ image is itself a unique and path-breaking way for compressing images. 


\subsection{D Spiral JPEG Encoding}

The flowchart for this method is illustrated in fig 4.1

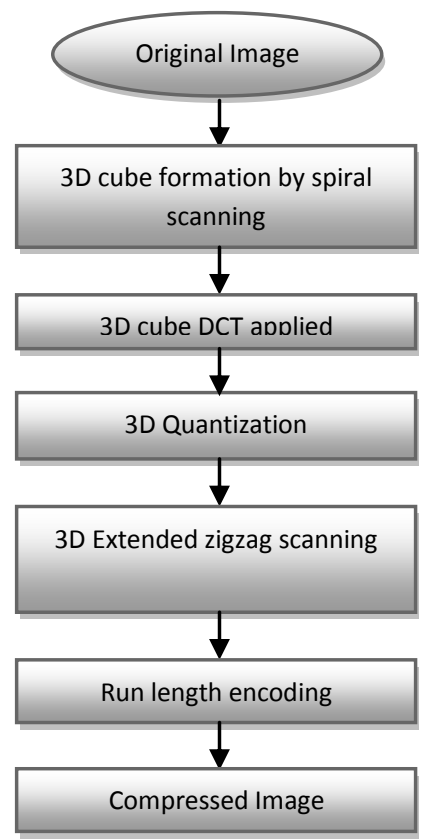

Fig. 4.1 Flowchart for compressing process

\subsection{Algorithm for 3D Spiral JPEG}

1. In this compression scheme, the 2-D image is initially divided into a set of $8 * 8$ pixel blocks. Then an $8 * 8 * 8$ dimensional 3-D cube is formed simply using spiral scanning procedure on each $8 * 8$ pixel block starting from the center of the graphic and going outwards as shown in Fig. 4.2.

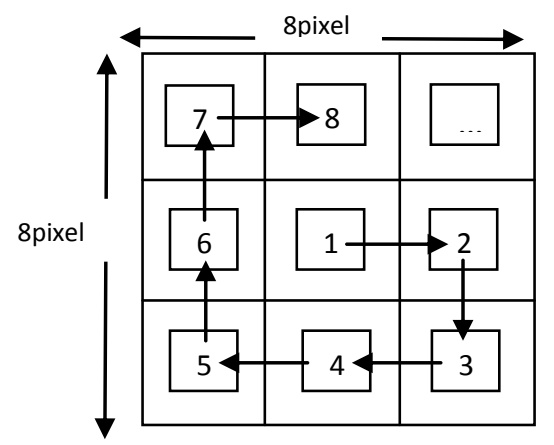

Fig.4.2 Spiral scanning of the image

2. This is done to the complete image in a set of $8 * 8$ blocks. Now each set of $8 * 8$ blocks is positioned on top of each other forming layered structure and assembled into a $3 \mathrm{D}$ data cube of $8 * 8 * 8$ pixels as shown in Fig.4.3.

Hence it becomes a three dimensional cube consisting of pixels and giving more detailed information about the image.
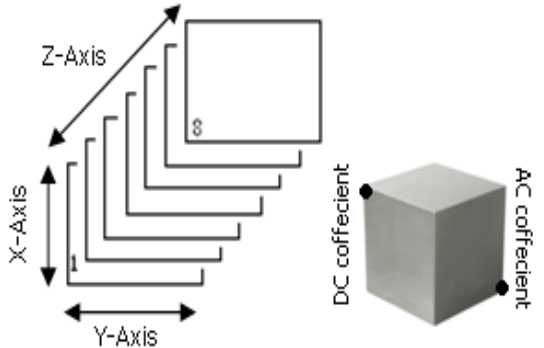

Fig $4.38 * 8 * 8$ cube formation

After implementation of the 3D data cubes arrangement, data values of each cube are level shifted. Then 3D-DCT is performed for each of 3D data cubes. Following the 3D-DCT, the DC coefficients are likely to be concerted nearer to the more important coefficients i.e. in upper left corner of the 3D cube and get diminished as they move towards the AC coefficient i.e. less significant ones in the lower right corner of the $3 \mathrm{D}$ cube. Since the general three-dimensional DCT core is distinguishable, the 3D DCT is generally obtained by applying the one-dimensional DCT along each of the 3-dimensions as follows:

$$
Z(l, m, n)=\sqrt{(8 / N 3) \sum \sum \sum x(i, j, k) C i j \text { Cjm Ckn }}
$$

Then, it is quantized using the predefined quantization table with the following formula:

$$
Q(x, y, z)=4 x+10 y+1 z
$$

The proposed quantization table can be multiplied by an integer value to get changing levels of compression rates and image quality.

3. Using the 3-D zigzag scanning the cube which was formed is converted into a $1 \mathrm{D}$ vector containing the data values of the figure containing information. In this method each pixel is scanned in zigzag manner along all the 3 axes; $\mathrm{x}$-axis, $y$ axis and z-axis.

4. By using this scan order, large runs of zeros are obtained which are compressed very well when run length encoding [7] is applied on it.

The decoding method is simply the reverse of the encoding method [2].

\section{RESULT ANALYSIS}

A comparative study of all the compression techniques implemented is done and the results are evaluated [8]. A particular picture depending upon its parameters can be compressed using various techniques but the one with the most efficient compression ratio and an acceptable image quality must be implemented. Different methods have different advantages and disadvantages based on computational time required or calculated PSNR, MSE etc. and other parameters.

The above explained compression algorithms are evaluated for the image shown in Fig. 5.1

The evaluated data is summarized in the Table 1 and Table 3 shown below whereas the computational time required for each 
technique is given in Table2. The best compression ratio is offered by the 3D Spiral Technique whereas DWT textured image compression offers an efficient PSNR (Peak Signal to Noise Ratio) value.

Table1: Performance On The Basis Of Compression offered by different Algorithms.

\begin{tabular}{|c|c|c|}
\hline Algorithm & PSNR value & Compression Ratio \\
\hline DWT & 34.66 & $>>35$ \\
\hline K-Means & 27.63 & $<25$ \\
\hline 3D Spiral JPEG & 31.73 & $<=60$ \\
\hline
\end{tabular}

The most competent and fastest compression technique is the DWT method, but the quality of compression is moderate as compared to the K-Means and 3D spiral compression Technique.

So when time is the parameter to be conserved DWT Technique should be implemented but when a more proficient compression has be to achieved 3D Spiral is the most preeminent.

Table 2 .Performance On The Basis of Compression Time offered by different algorithms

\begin{tabular}{|c|c|c|}
\hline Algorithm & $\begin{array}{c}\text { Encoding } \\
\text { Time(sec) }\end{array}$ & $\begin{array}{c}\text { Decoding } \\
\text { Time(sec) }\end{array}$ \\
\hline DWT & 2.8 & 1.7 \\
\hline K-Means & 4.5 & 3.0 \\
\hline 3D Spiral JPEG & 6.5 & 3.5 \\
\hline
\end{tabular}

While performing image compression, it is of great importance to know how much significant information can be made redundant from input data so as to maintain critical information of the original data. The various parameters compared [8] are mean square error, structural content, absolute error and after comparing these parameters a conclusion is summarized so that the most capable technique should be used. This is illustrated in Table3.

Table 3 .Comparison of different Image compression Techniques

\begin{tabular}{|c|c|c|c|}
\hline $\begin{array}{c}\text { Performance } \\
\text { parameter }\end{array}$ & DWT & $\begin{array}{c}\text { 3D } \\
\text { Spiral } \\
\text { JPEG }\end{array}$ & K-means \\
\hline Mean Square Error & 622.56 & $5.43^{*} \mathrm{e}^{3}$ & $1.0485^{*} \mathrm{e}^{3}$ \\
\hline S/N ratio & 20.18 & 10.7928 & 17.925 \\
\hline $\begin{array}{c}\text { Normalized Cross } \\
\text { correlation }\end{array}$ & 0.9891 & 0.9518 & 0.9809 \\
\hline Average Difference & -0.8819 & -9.5432 & 0.7268 \\
\hline Structural Content & 1.0027 & 0.9488 & 1.0092 \\
\hline Maximize Difference & 199 & 239 & 213 \\
\hline (Absolute Error) & 0.0716 & 0.2471 & 0.0719 \\
\hline
\end{tabular}

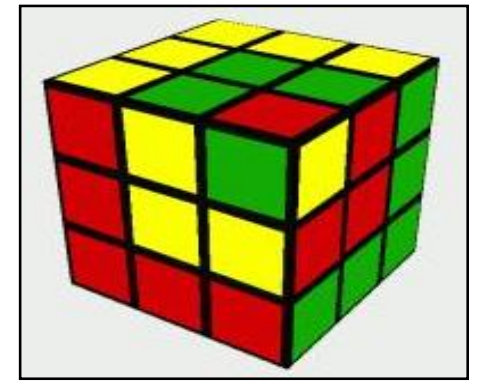

Fig.5.1. Original image of Cube ('icube.bmp')

The compressed images of the Cube based on the three approaches are shown in Fig.5.2

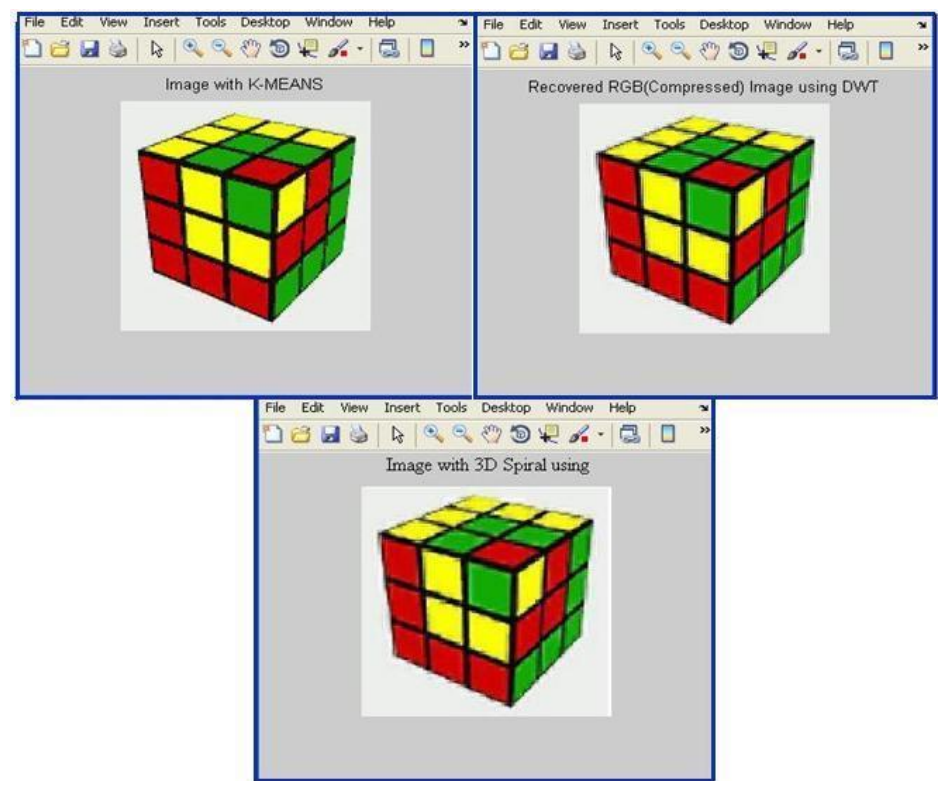

Fig.5.2 Compressed image of Cube by (a) K-MEANS (b) DWT, (c) 3D DCT Spiral

By comparing all the three image compression techniques, a conclusion can be derived that the Wavelet Transform Technique (DWT) can be used when there is a demand for a less compression time. The 3D Spiral JPEG Technique using DCT is the optimum algorithm where highest compression ratio is the necessitate [4]. However, the disadvantage in these algorithms is that there is coefficient quantization and bit allocation. Also in 3D Spiral technique the formation of cube and a $3 \mathrm{D}$ zigzag performed on the cube is a very slow process. Hence when it is required to be implemented on high resolution or high quality pictures it consumes a lot of time. Also when the Vector Quantization Technique is implemented, using K-mean algorithm, there is no coefficient quantization, which is a major advantage. However, the slow code book generation procedure and the small bits per pixel are the disadvantages. For faster compression and decompression, [7] DWT textured or the Vector quantized method should be used. 


\section{CONCLUSION}

This paper is therefore reviewed and summarized according to the individuality of three up-to-date image coding algorithms based on Discrete Wavelet Transform, 3D Spiral JPEG and Kmeans- a vector quantization technique. Experimental results using many standard color images authenticate the ability of the proposed scheme in achieving higher compression ratio while preserving good visual quality. Experimental comparisons on image of 'icube.bmp' in fig.5.1 recommend a recipe described as follows. The qualities in compressed images, including CR, PSNR, MSE and their encoding and decoding times were comprehensively examined in this study. Any of the three approaches is satisfactory when the 0.5 bits per pixel (bpp) is requested.

However, for a very low bit rate, for example 0.25 bpp or lower, the color embedding using discrete wavelet Transform (DWT) approach is superior [4] to other approaches. For practical applications, Wavelet based compression algorithms are recommended. 3D DCT based approach is suggested for images having large and common background information. It provides better performance than conventional JPEG images and where there is substantial amount of background. Vector Quantization approach using K-means is not appropriate for a low bit rate compression although it is simple and gives efficient results. It would be an ultimate option for time-bound applications online search engines of image and multimedia databases.

\section{ACKNOWLEDGEMENT}

We would like to express our gratitude towards our guide and Head of Department (H.O.D) Dr. Parul Shah for her crucial guidance, assistance in our project and for being a constant source of inspiration to us. We are also thankful to our institute Sardar Patel Institute of Technology, Mumbai, India for providing the amenities to carry out the research work.

\section{REFERENCES}

[1] Ricardo L. de Queiroz and Karen M.Braun, 'Color to Gray and Back: Color Embedded into Gray Images,' IEEE Transactions on Image Processing's vol. 15, pp. 1464-1467, June 2006.

[2] M. Alptekin Engin and Bulent Cavusoglu, 'New Approach in Image Compression: 3D Spiral JPEG,' IEEE Communications Letters vol. 15, no. 11, November 2011.

[3] Bang Huang; Linbo Xie, "An improved LBG algorithm for image vector quantization," Compute Science and Information Technology (ICCSIT), 2010 3rd IEEE International Conference on, vol.6, no., pp.467, 471, 911 July2010.

[4] Anil Kumar Katharotiya, Swati Patel, Mahesh Goyani, "Comparative Analysis between DCT \& DWT Techniques of Image Compression". Journal of Information Engineering and Applications, Vol. 1, No. 2, 2011.

[5] K Somasundaram and Mary Shanthi M Rani. Article: Novel K-means Algorithm for Compressing Images. International Journal of Computer Applications 18(8):9-13, March2011.

[6] Frederic Lehmann, "Turbo Segmentation of Textured Images," IEEE Transactions on Pattern Analysis and Machine Intelligence, vol. 33, no. 1, pp. 16-29, Jan. 2011.

[7] Harjeetpal Singh, Sakhi Sharma, "Hybrid Image Compression Using DWT, DCT \& Huffman Encoding Techniques", International Journal of Emerging Technology and Advanced Engineering, (ISSN 2250-2459, Volume 2, Issue 10,October2012.

[8] Kiran Bindu, Anita Ganpati, Aman Kumar Sharma, "A COMPARATIVE STUDY OF IMAGE COMPRESSION ALGORITHMS", International Journal of Research in Computer Science, eISSN 2249-8265 Volume 2 Issue 5 (2012)pp.37-42. 